\title{
Textbook Examples of Recursion
}

by Donald E. Knuth

Abstract. We discuss properties of recursive schemas related to McCarthy's "91 function" and to Takeuchi's triple recursion. Several theorems are proposed as interesting candidates for machine verification, and some intriguing open questions are raised.

John McCarthy and Ikuo Takeuchi introduced interesting recurrence equations as they were exploring the properties of recursive programs. McCarthy's function [7]

$$
f(x)=\text { if } x>100 \text { then } x-10 \text { else } f(f(x+11))
$$

has become known as the "91 function," since it turns out that $f(x)=91$ for all $x \leq 101$. Takeuchi's function [13] is a triple recursion

$$
t(x, y, z)=\text { if } x \leq y \text { then } y \text { else } t(t(x-1, y, z), t(y-1, z, x), t(z-1, x, y)),
$$

which has proved useful for benchmark testing of Lisp systems because the recursion terminates only after the definition has been expanded a large number of times (assuming that previously computed values are not remembered). Neither of these functions is of practical importance, because no reasonable programmer would ever want to carry out such recursive computations on a realistic problem. Yet both functions are quite instructive because they illustrate important problems and techniques that arise when we consider the task of verifying computer programs formally. Therefore they make excellent examples for textbooks that discuss recursion.

The purpose of this paper is to obtain new information about $f(x)$ and $t(x, y, z)$ and about several closely related functions. Several of the theorems proved below should provide good test material for automated verification systems. A few open problems are stated, illustrating the fact that extremely simple recursions can lead to quite difficult questions.

1. The 91 function. It is appropriate to begin by studying the 91 function, because 1991 is the year of John McCarthy's 64th birthday (and because a computer scientist's most significant birthday is the 64th). McCarthy originally wrote down the definition of $f(x)$, as shown above, because he wanted to study a simple recursion whose properties could not be deduced by ordinary mathematical induction. After studying the definition, he was pleasantly surprised to discover that it had the totally unexpected "91 property."

The 91 function certainly belongs to the set of significant textbook examples, because it is mentioned on at least 14 pages of Zohar Manna's well known text, Mathematical Theory of Computation [6]. The first published discussions of the function appeared in 1970 [7, 9], after it had been investigated extensively at Stanford's AI laboratory during 1968 [8].

Instead of using McCarthy's original definition, let's change the specifications a bit and consider the following function (see [6, Problem 5-8]):

$$
f(x)=\text { if } x>100 \text { then } x-10 \text { else } f^{91}(x+901)
$$


where $f^{91}(y)$ stands for $f(f(\cdots(f(y)) \cdots))$, the 91-times-repeated application of $f$. According to this new definition, we have

$$
f(91)=f^{91}(992)=f^{90}(982)=\cdots=f^{2}(102)=f(92) .
$$

And a similar derivation shows that if $90 \leq x \leq 100$ we have

$$
f(x)=f^{91}(x+901)=\cdots=f^{2}(x+11)=f(x+1)
$$

hence

$$
f(90)=f(91)=\cdots=f(100)=f(101) .
$$

And $f(101)=91$, so we have proved in particular that

$$
f(91)=91
$$

Now let's evaluate $f(x)$ when $x$ is extremely small, say $x=-10^{6}$. We have

$$
\begin{aligned}
f(-1000000)=f^{91}(-999099)=f^{181}(-998198)=\cdots & =f^{99811}(-791) \\
& =f^{99901}(110) \\
& =f^{99900}(100)
\end{aligned}
$$

and we know that $f(100)=91$; hence

$$
f^{99900}(100)=f^{99899}(91)=f^{99898}(91)=\cdots=f(91)=91 .
$$

In general, if $x$ is any integer $\leq 100$, let $m$ be the smallest integer such that $x+901 m>100$, and let $n$ be the smallest integer such that $x+901 m-10 n \leq 100$. Then $m \geq 1, n \leq 91$, and

$$
f(x)=f^{1+90 m}(x+901 m)=f^{1+90 m-n}(x+901 m-10 n)=f^{90 m-n}(91),
$$

where the last step follows since $91 \leq x+901 m-10 n \leq 100$. We conclude that $f(x)=91$. (The final step is omitted if $m=1$ and $n=91$; that case occurs if and only if $x=100$.)

How many iterations are needed to compute $f(x)$ by this definition, if we continue to apply the recurrence even when evaluating $f(x)$ for values of $x$ that have already been considered? Let $F(x)$ count the number of times that the test 'if $x>100$ ' is performed; then we have

$$
\begin{aligned}
& F(x)=\text { if } x>100 \text { then } 1 \text { else } 1+F(x+901)+F(f(x+901))+ \\
& \qquad+F\left(f^{2}(x+901)\right)+\cdots+F\left(f^{90}(x+901)\right) .
\end{aligned}
$$

(This is a special case of the general notion of a derived function, which is always jointly recursive with the function from which it has been derived; see McCarthy and Talcott [11].) A bit of experimentation reveals that $F(x)$ also reduces to a simple function: 
Lemma 1. $F(x)=$ if $x>100$ then 1 else $9192-91 x$.

Proof. If $x<100$ we have

$$
F(x)-F(x+1)=\sum_{k=0}^{90}\left(F\left(f^{k}(x+901)\right)-F\left(f^{k}(x+902)\right)\right) .
$$

Now if $x+901 \leq 100$, the sum reduces to $F(x+901)-F(x+902)$, because the terms for $k>0$ are $F\left(f^{k}(x+901)\right)-F\left(f^{k}(x+902)\right)=F(91)-F(91)=0$. In this case we let $x^{\prime}=x+901$. On the other hand if $x+901>100$, let $n$ be minimal such that $x+901-10 n \leq 100$. Then $1 \leq n \leq 90$, and $F\left(f^{k}(x+901)\right)-F\left(f^{k}(x+902)\right)=F(91)-F(91)=0$ for all $k>n$. We also have $F\left(f^{n}(x+901)\right)-F\left(f^{n}(x+902)\right)=F(x+901-10 n)-F(x+902-10 n)$; and $F\left(f^{k}(x+\right.$ $902))-F\left(f^{k}(x+901)\right)=1-1=0$ for all $k<n$. In this case we let $x^{\prime}=x+901-10 n$. In both cases we have found an $x^{\prime}$ such that

$$
F(x+1)-F(x)=F\left(x^{\prime}+1\right)-F\left(x^{\prime}\right), \quad x<x^{\prime} \leq 100 .
$$

The proof is therefore complete by induction on $101-x$ if we simply verify that $F(100)-F(101)=$ 91.

The 91 function suggests that we consider the more general recursive scheme

$$
f(x)=\text { if } x>a \text { then } x-b \text { else } f^{c}(x+d),
$$

where $a$ is an arbitrary real number, $b$ and $d$ are positive reals, and $c$ is a positive integer.

Theorem 1. The generalized 91 recursion with parameters $(a, b, c, d)$ defines a total function on the integers if and only if $(c-1) b<d$. In such a case the values of $f(x)$ also obey the much simpler recurrence

$$
f(x)=\text { if } x>a \text { then } x-b \text { else } f(x+d-(c-1) b) .
$$

Proof. It is not difficult to show that any function satisfying the generalized 91 recursion for $c>1$ must also satisfy

$$
f(x)=\text { if } x>a \text { then } x-b \text { else } f^{c-1}(x+d-b) .
$$

For if $x \leq a$, let $n$ be minimal such that $x+n d>a$. Then

$$
\begin{aligned}
& f^{c}(x+d)=f^{c+(n-1)(c-1)}(x+n d)=f^{n(c-1)}(x+n d-b) ; \\
& f^{c-1}(x+d-b)=f^{c-1+(n-1)(c-1)}(x+d-b+(n-1) d) ;
\end{aligned}
$$

hence $f^{c}(x+d)=f^{c-1}(x+d-b)$, as desired.

To complete the proof, we use induction on $c$; and we also need to characterize the parameter settings that cause the given recursive definition to terminate for all $x$.

If $(c-1) b \geq d$, the expansion of $f(x)$ will not terminate when $a-b<x \leq a$. For if $n$ is minimum such that $x+d-n b \leq a$, we have $n \leq c-1$, and

$$
f(x)=f^{c}(x+d)=\cdots=f^{c-n}(x+d-n b) .
$$


Now $c-n>0$, and $a-b<x+d-n b \leq a$, so this will go on and on.

On the other hand, we can show that no looping will occur if $(c-1) b<d$. Suppose first that $x>a-b$. If $x>a$, obviously $f(x)=x-b$. Otherwise we have $x+d>x+(c-1) b>a+(c-2) b$, hence

$$
f(x)=f^{c}(x+d)=\cdots=f^{2}(x+d-(c-2) b)=f(x+d-(c-1) b) .
$$

Let $\Delta=d-(c-1) b$, and let $m$ be minimum such that $x+m \Delta>a$; then

$$
f(x)=f(x+\Delta)=\cdots=f(x+m \Delta)=x+m \Delta-b .
$$

Thus, the expansion of $f(x)$ terminates with a value $>a-b$ whenever $x>a-b$.

Finally, if $x \leq a-b$ and if $m$ is minimal such that $x+m d>a-b$, the expansion of

$$
f(x)=f^{1+m(c-1)}(x+m d)
$$

terminates, because we can peel off the $f$ 's one by one.

When the generalized 91 function is total, we can express it in "closed form" as

$$
\begin{aligned}
f(x)= & \text { if } x>a \text { then } x-b \\
& \text { else } a+d-c b-((a-x) \bmod (d-(c-1) b)) .
\end{aligned}
$$

The special case $c=2$ of Theorem 1 was first proved by Manna and Pnueli [8].

Open Problem 1. Prove Theorem 1 by computer.

2. The Takeuchi function. Now we turn to the more complex recurrence

$$
t(x, y, z)=\text { if } x \leq y \text { then } y \text { else } t(t(x-1, y, z), t(y-1, z, x), t(z-1, x, y)) .
$$

John McCarthy observed in unpublished notes [10] that this function can be described more simply as

$$
t(x, y, z)=\text { if } x \leq y \text { then } y \text { else if } y \leq z \text { then } z \text { else } x .
$$

If we assume termination, the latter function satisfies Takeuchi's recurrence, so it must be identical with the former function.

John had just returned from a conference in Kyoto, and his notes [10] began with a brief comment about the history of this function and its motivation:

Ikuo Takeuchi (1978) of the Electrical Communication Laboratory of Nippon Telephone and Telegraph Co. (Japan's Bell Labs) devised a recursive function program for comparing the speeds of LISP systems. It can be made to run a long time without generating large numbers or using much stack.

(Incidentally, I believe [10] was John's first experiment with the use of $\mathrm{T}_{\mathrm{E}} \mathrm{X}$, a computer typesetting system that I was developing while sitting in the office next to his. Without his generous provision of computing and printing facilities, $\mathrm{T}_{\mathrm{EX}}$ would never have existed.) 
At about the same time, John coerced the FOL proof-checking system to construct a 50 -step proof that $A(x, y, z)$ has the simple form stated above [10]. This experiment suggested several improvements to FOL.

If we fully expand the definition of $t(x, y, z)$ whenever $x>y$, the proof of termination seems to be nontrivial, because there is no obvious way to impose an order on the set of all arguments $(x, y, z)$ in such a way that no infinitely long dependency chains exist. We shall prove termination as a byproduct of a more general investigation of the total running time needed to evaluate $t(x, y, z)$ by repeated application of the definition.

Let $T(x, y, z)$ be the number of times the else clause is invoked when $t(x, y, z)$ is evaluated by ordinary Lisp recursion. Then

$$
\begin{aligned}
T(x, y, z)= & \text { if } x \leq \\
\text { else } 1 & +T(x-1, y, z)+T(y-1, z, x)+T(z-1, x, y) \\
& +T(t(x-1, y, z), t(y-1, z, x), t(z-1, x, y)) .
\end{aligned}
$$

The total number of expansions of the definition will then be $1+4 T(x, y, z)$, because the latter function $\mathcal{T}(x, y, z)$ satisfies the recurrence

$$
\begin{aligned}
\mathcal{T}(x, y, z)=\text { if } x \leq & y \text { then } 1 \\
\text { else } 1 & +\mathcal{T}(x-1, y, z)+\mathcal{T}(y-1, z, x)+\mathcal{T}(z-1, x, y) \\
& +\mathcal{T}(t(x-1, y, z), t(y-1, z, x), t(z-1, x, y)) .
\end{aligned}
$$

Before we analyze $T(x, y, z)$ it will be helpful to consider a similar but simpler function

$$
\begin{aligned}
V(x, y, z)= & \text { if } x \leq y \text { then } 0 \\
& \text { else } 1+V(x-1, y, z)+V(y-1, z, x)+V(z-1, x, y) .
\end{aligned}
$$

The function $V(x, y, z)$ can be understood as follows. Construct a ternary tree by starting with a simple leaf containing the triple $[x, y, z]$ and repeatedly applying the following operation: If any leaf $[x, y, z]$ of the tree-so-far has $x>y$, attach the nodes

$$
[x-1, y, z], \quad[y-1, z, x], \quad[z-1, x, y]
$$

immediately below it. Then $V(x, y, z)$ will be the number of nonleaf nodes in the final tree. (This function $V(x, y, z)$ has been studied by Ilan Vardi [14]; some of his analysis is reproduced here.)

[Note: I have an example in the MS., but I'll skip it unless I get goahead from Vlad later.]

The evaluation of $V(x, y, z)$ is trivial when $x \leq y$, and it's also fairly simple when $x>y$ and $x \geq z \geq y$ : In that case we have

$$
V(x, y, z)=1+V(x-1, y, z)=1+x-z+V(z-1, y, z) .
$$

A further simplification arises when we realize that the values of $V(x, y, z)$ are invariant if we translate all the parameters by any integer amount:

$$
V(x+1, y+1, z+1)=V(x, y, z) .
$$


Therefore we can shorten our notation and our discussion by assuming that $\min (x, y, z)=0$.

Suppose the ternary tree has $[x, y, z]$ at the root, where $\min (x, y, z)=0$ and either $x>y>z$ or $z>x>y$. Then all of its non-leaf nodes are of two kinds,

$$
\begin{aligned}
& A(a, b)=[a, b, 0] \quad \text { where } a>b>0 . \\
& B(a, b)=[b, 0, a] \quad
\end{aligned}
$$

Below $A(a, b)$ are the three nodes

$$
[a-1, b, 0], \quad[b-1,0, a], \quad[-1, a, b]
$$

where $[a-1, b, 0]$ is a leaf if $a=b+1$, otherwise it is $A(a-1, b)$; similarly $[b-1,0, a]$ is a leaf if $b=1$, otherwise it is $B(a, b-1)$; and $[-1, a, b]$ is always a leaf. Below $B(a, b)$ are the same three nodes; they appear in a different order, but that does not matter.

It follows that $V(x, y, 0)=V(y, 0, x)$, for all $x>y>0$, and that $V(x, y, 0)$ has a simple combinatorial interpretation: It is the number of lattice paths that start at $(x, y)$ and stay within the set $\{(a, b) \mid a>b>0\}$. (A lattice path is a path in which each step decreases exactly one of the coordinates by unity.) We will say that such a lattice path is confined.

Confined lattice paths can be enumerated by using André's well-known reflection principle (see, for example, [4, exerise 2.2.1-4]). Given $x>y \geq y^{\prime}>0$, the number of confined paths from $(x, y)$ to a point $\left(x^{\prime}, y^{\prime}\right)$ for some $x^{\prime}$ is equal to the number of all possible lattice paths from $(x, y)$ to $\left(y^{\prime}, y^{\prime}-1\right)$ minus the number of such paths that touch a diagonal point. Paths of the latter type are in one-to-one correspondence with lattice paths from $(x, y)$ to $\left(y^{\prime}-1, y^{\prime}\right)$; the correspondence is obtained by interchanging $x$ moves with $y$ moves after the diagonal is first encountered. Hence the number of confined paths from $(x, y)$ to points of the form $\left(x^{\prime}, y^{\prime}\right)$, given $x, y$, and $y^{\prime}$, is

$$
\left(\begin{array}{c}
x-y^{\prime}+y-\left(y^{\prime}-1\right) \\
x-y^{\prime}
\end{array}\right)-\left(\begin{array}{c}
x-\left(y^{\prime}-1\right)+y-y^{\prime} \\
y-y^{\prime}
\end{array}\right)
$$

and the total number of confined paths starting from $(x, y)$ is

$$
\begin{aligned}
\sum_{y^{\prime}=1}^{y} & \left(\left(\begin{array}{c}
x+y+1-2 y^{\prime} \\
x-y^{\prime}
\end{array}\right)-\left(\begin{array}{c}
x+y+1-2 y^{\prime} \\
y-y^{\prime}
\end{array}\right)\right) \\
& =\sum_{k=1}^{y}\left(\left(\begin{array}{c}
x-y-1+2 k \\
k
\end{array}\right)-\left(\begin{array}{c}
x-y-1+2 k \\
k-1
\end{array}\right)\right) .
\end{aligned}
$$

This is the quantity $V(x, y, 0)$. Notice that we have

$$
V(n+1, n, 0)=\sum_{k=1}^{n}\left(\left(\begin{array}{c}
2 k \\
k
\end{array}\right)-\left(\begin{array}{c}
2 k \\
k-1
\end{array}\right)\right)=\sum_{k=1}^{n}\left(\begin{array}{c}
2 k \\
k
\end{array}\right) \frac{1}{k+1}=\sum_{k=1}^{n} C_{k},
$$

the sum of the first $n$ Catalan numbers.

Returning to the evaluation of $T(x, y, z)$, we must add to $V(x, y, z)$ the values $T(t(a-1, b, 0)$, $t(b-1,0, a), a)$ at every node of type $A(a, b)$, and the values $T(t(b-1,0, a), a, t(a-1, b, 0))$ at every 
node of type $B(a, b)$. Fortunately these additional amounts are mostly zero. Our knowledge about the values of $t(x, y, z)$ allows us to conclude that, when $a>b>0$, we have

$$
t(a-1, b, 0)=a-1 ; \quad t(b-1,0, a)= \begin{cases}0, & b=1 \\ a, & b>1\end{cases}
$$

Therefore $T(t(b-1,0, a), a, t(a-1, b, 0))=0 ;$ and $T(t(a-1, b, 0), t(b-1,0, a), a)=0$ except when $b=1$. Only the nodes of type $A(a, 1,0)$ acquire additional values; and at such nodes we add $T(a-1,0, a)$.

The number of non-root nodes of type $A\left(a^{\prime}, 1\right)$ in a tree whose root is of type $A(a, b)$ or $B(a, b)$ is the number of nodes of type $A\left(a^{\prime}+1,1\right)$ or $B\left(a^{\prime}+1,1\right)$. And the number of such nodes is the number of confined lattice paths from $(a, b)$ to $\left(a^{\prime}+1,1\right)$, which is

$$
\left(\begin{array}{c}
a-\left(a^{\prime}+1\right)+b-1 \\
b-1
\end{array}\right)-\left(\begin{array}{c}
a-1+b-\left(a^{\prime}+1\right) \\
a-1
\end{array}\right)
$$

by André's reflection principle. Therefore

$$
T(b, 0, a)=V(a, b, 0)+\sum_{a^{\prime}=2}^{a-1}\left(\left(\begin{array}{c}
a+b-a^{\prime}-2 \\
b-1
\end{array}\right)-\left(\begin{array}{c}
a+b-a^{\prime}-2 \\
a-1
\end{array}\right)\right) T\left(a^{\prime}-1,0, a^{\prime}\right) .
$$

The same formula holds for $T(a, b, 0)$, except that we must add $T(a-1,0, a)$ when $b=1$; a root node of type $A(a, 1)$ makes a contribution, but a root node of type $B(a, 1)$ does not. Putting these facts together yields the following recurrence for the numbers $T_{n}=T(n, 0, n+1)$ :

$$
T_{n+1}=V(n+2, n+1,0)+\sum_{k=0}^{n-1}\left(\left(\begin{array}{c}
n+k \\
n
\end{array}\right)-\left(\begin{array}{c}
n+k \\
n+1
\end{array}\right)\right) T_{n-k} .
$$

Let $V_{n}=V(n+1, n, 0)$; the first few values of these sequences are as follows:

$$
\begin{array}{rrrrrrrrrr}
n=1 & 2 & 3 & 4 & 5 & 6 & 7 & 8 & 9 \\
V_{n}=1 & 3 & 8 & 22 & 64 & 196 & 625 & 2055 & 6917 \\
T_{n}=1 & 4 & 14 & 53 & 223 & 1034 & 5221 & 28437 & 165859
\end{array}
$$

It is not difficult to deduce that the numbers $T_{n}$ grow very rapidly, in fact faster than $A^{n}$ for any constant $A$ :

Lemma 2. If $\epsilon>0$, we have

$$
T_{n}>n^{(1-\epsilon) n}
$$

for all sufficiently large $n$.

Proof. Choose $k$ large enough so that $k /(k+1)>1-\epsilon$. Looking only at the $k$ th term of the recurrence for $T_{n}$ tells us that, for all $n>k$, we have

$$
T_{n+1}>\left(\left(\begin{array}{c}
n+k \\
n
\end{array}\right)-\left(\begin{array}{c}
n+k \\
n+1
\end{array}\right)\right) T_{n-k}=\frac{(n+k)(n+k-1) \ldots(n+2)(n+1-k)}{k !} T_{n-k} .
$$


Thus

$$
\ln T_{n+1}>\ln (n+k)+\cdots+\ln (n+2)+\ln (n+1-k)-\ln k !+\ln T_{n-k} .
$$

Iterating this relation yields

$$
\ln T_{n}>\frac{k}{k+1} n \ln n+O(n)
$$

and the result follows. (A similar but weaker result was obtained by Ilan Vardi [14], who used the fact that $T_{n+1}>n T_{n-1}$ to prove that $\ln T_{n}>\frac{1}{2} n \ln n+O(n)$.)

In fact, we can prove a stronger lower bound by observing that for all $n \geq 1$, we have $T_{n} \geq b_{n}$, where $b_{n}$ is the Bell number defined by

$$
b_{n+1}=1+\sum_{k=0}^{n-1}\left(\begin{array}{l}
n \\
k
\end{array}\right) b_{n-k} .
$$

Each term in the recurrence for $T_{n}$ is greater than or equal to the corresponding term in the recurrence for $b_{n}$, by induction. It is known $[1,(6.2 .7)]$ that

$$
b_{n}>e^{n \ln n-n \ln \ln n-n}
$$

for all sufficiently large $n$. Thus $T_{n}$ grows faster than $(n / e \ln n)^{n}$.

On the other hand, we can prove the upper bound

$$
T_{n}<3 n !
$$

This is clear for $n \leq 3$. Let $t_{n}=T_{n} / n$ !, and rewrite the recurrence for $T_{n}$ as follows:

$$
\begin{aligned}
t_{n+1}=\frac{V_{n+1}}{(n+1) !}+\frac{1}{n+1}\left(t_{n}\right. & +t_{n-1}+\frac{n+2}{2 n} t_{n-2} \\
& \left.+\frac{n+3}{3(n-1)} \frac{n+2}{2 n} t_{n-3}+\frac{n+4}{4(n-2)} \frac{n+3}{3(n-1)} \frac{n+2}{2 n} t_{n-4}+\cdots\right) .
\end{aligned}
$$

The coefficients inside the parentheses are all $\leq 1$, so we have

$$
t_{n+1} \leq \frac{V_{n+1}}{(n+1) !}+\frac{1}{n+1}\left(t_{n}+t_{n-1}+\cdots+t_{1}\right)<\frac{3 n}{n+1}+\frac{V_{n+1}}{(n+1) !}
$$

by induction. And it is easy to verify that $V_{n+1}<3 n$ ! for $n \geq 3$, because $V_{n+1} \leq 4^{n}$. (The Catalan numbers $C_{n}$ satisfy $C_{n+1}<4 C_{n}$, hence $V_{n+1}<C_{1}+4 V_{n}$ and we have $V_{n+1} \leq 4 V_{n}$.) We have proved

Theorem 2. When the Takeuchi recursion $t(x, y, z)$ is used in a memoryless manner to evaluate $t(n, 0, n+1)$, the definition is expanded $1+4 T(n, 0, n+1)$ times, where

$$
e^{n \ln n-n \ln \ln n-n}<T(n, 0, n+1)<e^{n \ln n-n+\ln n}
$$


for all sufficiently large $n$.

Only $O\left(n^{2}\right)$ evaluations are needed when previously computed results are remembered; thus memory is especially helpful here.

A more precise asymptotic formula for $V_{n}$ can be obtained from the well-known generating function for Catalan numbers [3, page 203],

$$
\begin{aligned}
V(z)=\sum_{n \geq 1} V_{n} z^{n} & =\frac{1}{1-z} \sum_{n \geq 1} C_{n} z^{n}=\frac{C(z)-1}{1-z}, \\
C(z) & =\frac{1-\sqrt{1-4 z}}{2 z} .
\end{aligned}
$$

Darboux's lemma (see [5]) now shows that

$$
\frac{V_{n}}{4^{n}}=\left[z^{n}\right] V\left(\frac{1}{4} z\right)=-\frac{8}{3}\left(\begin{array}{c}
n-3 / 2 \\
n
\end{array}\right)+O\left(n^{-5 / 2}\right)=\frac{4 n^{-3 / 2}}{3 \sqrt{\pi}}+O\left(n^{-5 / 2}\right) .
$$

The generating function of the numbers $T_{n}$ satisfies a remarkable functional equation: We have

$$
\begin{aligned}
T(z) & =\sum_{n} T_{n} z^{n} \\
& =\sum_{n} V_{n+1} z^{n+1}=\sum_{n, k}\left(\begin{array}{c}
n+k \\
k
\end{array}\right) T_{n-k} z^{n+1}-\sum_{n, k}\left(\begin{array}{c}
n+k \\
k-1
\end{array}\right) T_{n-k} z^{n+1} \\
& =\frac{C(z)-1}{1-z}+\sum_{n, k}\left(\begin{array}{c}
n+2 k \\
k
\end{array}\right) T_{n} z^{n+k+1}-\sum_{n, k}\left(\begin{array}{c}
n+2 k \\
k-1
\end{array}\right) T_{n} z^{n+k+1} \\
& =\frac{C(z)-1}{1-z}+\sum_{n} T_{n} z^{n+1} \frac{C(z)^{n}}{\sqrt{1-4 z}}-\sum_{n} T_{n} z^{n+2} \frac{C(z)^{n+2}}{\sqrt{1-4 z}} \\
& =\frac{C(z)-1}{1-z}+\frac{z(2-C(z))}{\sqrt{1-4 z}} T(z C(z))
\end{aligned}
$$

because $z C(z)^{2}=C(z)-1$ and $\sum_{k}\left(\begin{array}{c}n+2 k \\ k\end{array}\right) z^{k}=C(z)^{k} / \sqrt{1-4 z}$.

Open Problem 2. Obtain further information about the asymptotic properties of the coefficients $T_{1}, T_{2}, \ldots$.

The evaluation of $t(x, y, z)$ turns out to be much, much faster if we apply the technique of lazy evaluation or "call by need" when expanding the definition. (See Vuillemin [15], [16].) Indeed, we can ignore the third argument $t(z-1, x, y)$ in the recursion, unless we have discovered that $t(x-1, y, z)>t(y-1, z, x)$; so the number of times the else clause needs to be expanded satisfies the recursion

$$
\begin{aligned}
& K(x, y, z)=\text { if } x \geq y \text { then } 0 \\
& \begin{aligned}
\text { else }( & +K(x-1, y, z)+K(y-1, z, x) \\
+ & \text { if } t(x-1, y, z) \leq t(y-1, z, x) \text { then } 0 \\
\quad & \quad \text { else } K(z-1, x, y)+K(t(x-1, y, z), t(y-1, z, x), t(z-1, x, y))) .
\end{aligned}
\end{aligned}
$$


And this recursion turns out to be quite simple. First, if $x>y \leq z$, we have

$$
K(x, y, z)=1+K(x-1, y, z)=x-y
$$

because $t(x-1, y, z) \leq z$ and $t(y-1, z, x)=z$. Second, if $x>y>z+1$, we have

$$
\begin{aligned}
K(x, y, z) & =1+K(x-1, y, z)+K(y-1, z, x) \\
& =1+K(x-1, y, z)+y-1-z=(x-y)(y-z),
\end{aligned}
$$

because $t(x-1, y, z)=x-1$ and $t(y-1, z, x)=x$. Finally, if $x>y=z+1$, we have $t(x-1, y, z)=$ $x-1, t(y-1, z, x)=z$, and $t(z-1, x, y)=x$; hence

$$
K(x, y, z)=1+K(x-1, y, z)+x-1-z=(x-y)(x-y+3) / 2 .
$$

Incidentally, when $x>y>z+1$, the expansions of else clauses occur at the arguments $(\xi, y, z)$ and $(\eta, z, \xi)$ for $x \geq \xi>y$ and $y>\eta>z$; when $x>y=z+1$, they occur at $(\xi, y, z)$ and $(\eta, z, \xi)$ for $x \geq \xi>\eta \geq y$. Since these arguments are distinct, no additional savings over call-by-need would be obtained by remembering previously computed values, unless $t(x, y, z)$ is being evaluated at more than one point $(x, y, z)$. The fact that the necessary arguments are limited underlies the simple mechanical proof of termination found by Moore [12].

3. False Takeuchi functions. Vardi [14] has considered a general recursion scheme of the form

$$
v_{h}(x, y, z)=\text { if } x \leq y \text { then } h(x, y, z) \text { else } v_{h}\left(v_{h}(x-1, y, z), v_{h}(y-1, z, x), v_{h}(z-1, x, y)\right) .
$$

If we set $h(x, y, z)=0$, the function $v_{h}(x, y, z)$ will of course be identically zero; we will deduce that its value is zero after expanding the definition exactly $1+4 V(x, y, z)$ times, where $V(x, y, z)$ is the function considered in the previous section. This is clearly the minimum number of expansions necessary over all possible auxiliary functions $h(x, y, z)$, if we do not or cannot use call-by-need.

Richard Gabriel used a Takeuchi-like function in extensive benchmark tests of Lisp compilers, but his function was slightly different from Takeuchi's original:

$$
g(x, y, z)=\text { if } x \leq y \text { then } z \text { else } g(g(x-1, y, z), g(y-1, z, x), g(z-1, x, y)) .
$$

(Notice that in this case call-by-need is inapplicable.) Gabriel explains the discrepancy as follows [2, pp. 10-11]:

When the Computer Science Department at Stanford University obtained the first two or three Xerox Dolphins, John McCarthy asked me to do a simple benchmark test with him. We sat down, and he tried to remember the Takeuchi function, which had had wide circulation. Because it was simple and because there were many results for it in the literature, he felt that it would be a good initial test. Of course, John misremembered the function. But we did not realize it until I had gathered a great many numbers for it.

Indeed, Gabriel's book [2] gives detailed timings for the computation of $g(18,12,6)=7$ on 132 different configurations, and he lists four additional variants of $g$ that provide further types of benchmark tests. 
The seemingly trivial change from $t(x, y, z)$ to $g(x, y, z)$ actually makes $g(x, y, z)$ substantially easier to compute, if $t(x, y, z)$ is not evaluated with memory of previous results or with call-by-need. Vardi [14] has shown that the corresponding running time $G(n, 0, n+1)$ is asymptotically less than $(3+\sqrt{8})^{n}$, although the exact order of growth is not known. Vardi has also observed that Gabriel's recursion defines the following curious pattern of values:

$$
\begin{gathered}
g(x, y, z)=\text { if } x \leq y \text { then } z \\
\text { else if } y \geq z \text { then } \\
\text { if } y=z \text { or }(x-y) \text { odd then } y \\
\text { else } z+1 \\
\text { else if } z \leq x+1 \text { and }(z \leq x \text { or } x>y+1) \text { then } y \\
\text { else if }(z-x) \text { even then } x \\
\text { else } y+1 .
\end{gathered}
$$

Here is another example of a generalized Takeuchi recurrence whose solution exhibits odd-even behavior:

$$
\begin{aligned}
b(x, y, z)= & \text { if } x \leq y \text { then if } x=y=z \text { then } 0 \text { else } 1 \\
& \text { else } b(b(x-1, y, z), b(y-1, z, x), b(z-1, x, y)) .
\end{aligned}
$$

This time the output of the function is boolean - always either 0 or 1 -although $x, y, z$ range over all integers. The computed values turn out to be

$$
\begin{aligned}
b(x, y, z)= & \text { if } x \leq y \text { then if } x=y=z \text { then } 0 \text { else } 1 \\
& \text { else if } z>y+1 \text { then if }(x-z) \text { odd then } 0 \text { else } 1 \\
& \text { else if } y=z \text { then if }(x-y) \text { even then } 0 \text { else } 1 \\
& \text { else if }(x-y) \text { odd then } 0 \text { else } 1 .
\end{aligned}
$$

The generalized recursion $v_{h}$ does not always define a total function by repeated expansion. For example, consider the auxiliary function

$$
e(x, y, z)=\text { if } x \text { odd then } 0 \text { else } 1
$$

then we get

$$
v_{e}(1,0,0)=v_{e}\left(v_{e}(0,0,0), v_{e}(-1,0,1), v_{e}(-1,1,0)\right)=v_{e}(1,0,0)
$$

and the recursion loops endlessly. There is a simple characterization of the cases where $v_{h}$ is total in the boolean case:

Lemma 4. Let $h(x, y, z)$ map arbitrary integers $x, y, z$ into 0 or 1 . Then the recursive equation for $v_{h}(x, y, z)$ defines a total function $v_{h}$ except in the following three cases:

(i) $h(0,0,0)=1$ and $h(-1,0,1)=h(-1,1,0)=0$;

(ii) $h(0,0,1)=h(0,1,0)=1$ and $h(-1,1,1)=0$;

(iii) $h(0,0,0)=h(0,0,1)=h(-1,1,0)=1$ and $h(-1,0,1)=h(-1,1,1)=0$.

Proof. If $h(0,0,0)=0$ or $h(-1,0,1)=1$, we have the well-defined result

$$
v_{h}(1,0,0)=h(h(0,0,0), h(-1,0,1), h(-1,1,0)) ;
$$


otherwise we have

$$
v_{h}(1,0,0)=v_{h}(1,0, h(-1,1,0))
$$

which loops in case (i) but gives $v_{h}(1,0,0)=v_{h}(1,0,1)$ otherwise. Similarly, we find that $h(0,0,1)=$ 0 or $h(-1,1,1)=1$ implies

$$
v_{h}(1,0,1)=h(h(0,0,1), h(-1,1,1, h(0,1,0)) ;
$$

hence $v_{h}(1,0,1)$ is well defined whenever case (ii) does not hold, except in the case when it leads to $v_{h}(1,0,1)=v_{h}(1,0,0)$.

If neither case (i) nor case (ii) holds, then $v_{h}(x, y, z)$ is well defined for all boolean values $x, y, z$, except in case (iii). And when $v_{h}(x, y, z)$ is defined for all boolean $x, y, z$, we can evaluate $v_{h}(x, y, z)$ for all $x, y, z$ in $O(V(x, y, z))$ steps.

When the boolean function $v_{h}$ of Lemma 3 isn't total, we can always complete it to a total function $v_{h}(x, y, z)$ that does satisfy the recurrence. We simply assign arbitrary boolean values to $v_{h}(1,0,0)$ and/or $v_{h}(1,0,1)$, whichever is undefined. For example, there are four total functions

$v_{e}(x, y, z)$ that satisfy the recurrence arising from the auxiliary function $e(x, y, z)$ considered above:

$$
\begin{aligned}
v_{k}^{00}(x, y, z)= & \text { if } x \text { odd then } 0 \text { else } 1 \\
v_{e}^{01}(x, y, z)= & \text { if } x \leq y \text { then if } x \text { odd then } 0 \text { else } 1 \\
& \text { else if } x \text { even then } 1 \\
& \text { else if } y \text { odd then } 0 \\
& \text { else if } z \text { odd then } 1 \text { else } 0 ; \\
v_{e}^{10}(x, y, z)= & \text { if } x \leq y \text { then if } x \text { odd then } 0 \text { else } 1 \\
& \text { else if } x \text { odd then } \\
& \text { if } y \text { odd or } z \text { odd then } 0 \text { else } 1 \\
& \text { else if } y \text { odd or } z \text { odd then } 1 \\
& \text { else if } y \leq z \leq x \text { then } 1 \text { else } 0 ; \\
& \text { if } x \leq y \text { then if } x \text { odd then } 0 \text { else } 1 \\
& \text { else if } x \text { even then } 1 \\
& \text { else if } y \text { odd then } 0 \\
& \text { else if } z \leq y \text { then } 1 \\
& \text { else if } z \text { odd then } 1 \text { else } 0 .
\end{aligned}
$$

However, the recurrence $v_{h}(x, y, z)$ cannot be completed to a total function for arbitrary auxiliary functions $h(x, y, z)$. Consider, for example, the (admittedly contrived) mapping

$$
h(x, y, z)=2 x y-4 x+y+z-1 .
$$

There is no total function $v_{h}$ because we would otherwise have

$$
\begin{aligned}
v_{h}(2,1,4) & =v_{h}\left(h(1,1,4), h(0,4,2), v_{h}(h(2,2,1), h(1,1,3), h(0,3,2))\right) \\
& =v_{h}\left(2,5, v_{h}(2,1,4)\right)=16+v_{h}(2,1,4) .
\end{aligned}
$$


An incompletable function can even be constructed when we restrict ourselves to auxiliary functions that are limited by the condition

$$
h(x, y, z) \leq \max (x, y, z) .
$$

For example, suppose we define

$$
\begin{aligned}
h(x, y, z)= & \text { if }(x, y, z)=(1,1,4) \text { then } 4 \\
& \text { else if }(x, y, z)=(3,3,3) \text { then } 2 \\
& \text { else if }(x, y)=(2,3) \text { then } 1 \\
& \text { else if } \max (x, y, z) \geq 3 \text { then } 3 \\
& \text { else } \max (x, y, z) .
\end{aligned}
$$

Then we have $v_{h}(x, y, 3)=3$ whenever $3>x \geq y$. For if $x=y$, clearly $v_{h}(y, y, 3)=h(y, y, 3)=3$; otherwise

$$
\begin{aligned}
v_{h}(x, y, 3) & =v_{h}\left(v_{h}(x-1, y, 3), v_{h}(y-1,3, x), v_{h}(2, x, y)\right) \\
& =v_{h}(3,3, \leq 2)=3 .
\end{aligned}
$$

Therefore if $y<3$ we have

$$
\begin{aligned}
v_{h}(3, y, 3) & =v_{h}\left(v_{h}(2, y, 3), v_{h}(y-1,3,3), v_{h}(2,3, y)\right) \\
& =v_{h}(3,3,1)=3 .
\end{aligned}
$$

It follows that

$$
v_{h}(3, y, 3)=\text { if } y=3 \text { then } 2 \text { else } 3 .
$$

But we also must have

$$
\begin{aligned}
v_{h}(4,3,1) & =v_{h}\left(v_{h}(3,3,1), v_{h}(2,1,4), v_{h}(0,4,3)\right) \\
& =v_{h}\left(3, v_{h}\left(v_{h}(1,1,4), v_{h}(0,4,2), v_{h}(3,2,1)\right), 3\right) \\
& =v_{h}\left(3, v_{h}\left(4,3, v_{h}\left(v_{h}(2,2,1), v_{h}(1,1,3), v_{h}(0,3,2)\right)\right), 3\right) \\
& =v_{h}\left(3, v_{h}\left(4,3, v_{h}(2,3,3)\right), 3\right) \\
& =v_{h}\left(3, v_{h}(4,3,1), 3\right) .
\end{aligned}
$$

And there is no $y$ such that $y=v_{h}(3, y, 3)$.

Open Problem 3. If we restrict $h(x, y, z)$ to be strictly less than $\max (x, y, z)$, is there always a total function $v_{h}(x, y, z)$ that satisfies the generalized Takeuchi recurrence?

We have considered Takeuchi's special case $h(x, y, z)=y$ as well as Gabriel's special case $h(x, y, z)=z$, so it is natural to consider also the recurrence with $h(x, y, z)=x$. Let

$$
\begin{aligned}
k(x, y, z)= & \text { if } x \leq y \text { then } x \\
& \text { else } k(k(x-1, y, z), k(y-1, z, x), k(z-1, x, y)) .
\end{aligned}
$$

This recursive definition yields only a partial function because, for example, we have

$$
k(x+1, x, x)=k(x, x-1, x-1)=k(x-1, x-2, x-2)=\cdots .
$$

However, there are infinitely many ways to define a total function that does satisfy the $k$ recurrence: 
Theorem 3. Let $c$ be any integer. The function

$$
\begin{aligned}
k_{c}(x, y, z)= & \text { if } x \leq y \text { then } x \\
& \text { else if } y \leq z+1 \text { then } c \text { else } \min (y, c)
\end{aligned}
$$

satisfies the generalized Takeuchi recurrence stated above for $k(x, y, z)$.

Proof. Notice that we have the special values

$$
\begin{aligned}
& k_{c}(x, c, z)=\min (x, c) ; \\
& \text { if } x>y \text { then } k_{c}(x, y, c)=c .
\end{aligned}
$$

The proof is now by induction on $x-y$.

If $x=y+1$ we have

$$
k_{c}\left(k_{c}(x-1, y, z), k_{c}(y-1, z, x), k_{c}(z-1, x, y)\right)=k_{c}\left(y, k_{c}(y-1, z, y+1), k_{c}(z-1, y+1, y)\right)
$$

If $y \leq z+1$, this reduces to

$$
k_{c}(y, y-1, z-1 \text { or } c)=c
$$

and if $y \geq z+2$, it is

$$
k_{c}\left(y, c, k_{c}(z-1, y+1, y)\right)=\min (y, c) .
$$

Thus we obtain $k_{c}(x, y, z)$ when $x=y+1$.

If $x \geq y+2$ and $y \leq z+1$ we have

$$
k_{c}\left(k_{c}(x-1, y, z), k_{c}(y-1, z, x), k_{c}(z-1, x, y)\right)=k_{c}(c, y-1, z-1 \text { or } c \text { or } x) \text {, }
$$

which equals $c$ since $y-1 \leq z-1+1$ and $y-1 \leq x+1$.

And finally if $x \geq y+2$ and $y \geq z+2$ the right side of the recurrence reduces to

$$
k_{c}(\min (y, c), c, z-1)=\min (y, c) .
$$

Corollary. The least fixed point of the recursive definition $k(x, y, z)$ is if $x \leq y$ then $x$ else $\omega$. Proof. Whenever $x>y$, we have $k_{c}(x, y, z)=y$ when $c=y$ but not when $c<y$.

4. The Takeuchi recurrence in higher dimensions. If we define

$$
\begin{aligned}
t(w, x, y, z)= & \text { if } w \leq x \text { then } x \\
& \text { else } t(t(w-1, x, y, z), t(x-1, y, z, w), t(y-1, z, w, x), t(z-1, w, x, y))
\end{aligned}
$$

it turns out that the function reduces to the simple mapping

$$
\begin{aligned}
t(w, x, y, z)= & \text { if } w \leq x \text { then } x \text { else if } x \leq y \text { then } y \\
& \text { else if } y \leq z \text { then } z \text { else } w .
\end{aligned}
$$


Therefore it is natural to conjecture that the $m$-dimensional generalization

$$
\begin{aligned}
t\left(x_{1}, x_{2}, \ldots, x_{m}\right)= & \text { if } x_{1} \leq x_{2} \text { then } x_{2} \\
& \text { else } t\left(t\left(x_{1}-1, x_{2}, \ldots, x_{m}\right), \ldots, t\left(x_{m}-1, x_{1}, \ldots, x_{m-1}\right)\right)
\end{aligned}
$$

is satisfied by the $m$-dimensional "first rise" function

$$
\begin{aligned}
u\left(x_{1}, x_{2}, \ldots, x_{m}\right)= & \text { if } x_{1}>\cdots>x_{k} \leq x_{k+1} \text { for some } k \geq 1 \text { then } x_{k+1} \\
& \text { else } x_{1} .
\end{aligned}
$$

But this is false, for all $m>4$. Indeed, we have a 5-dimensional counterexample,

$$
\begin{aligned}
t(5,3,2,0,1) & =t(t(4,3,2,0,1), 2, t(1,0,1,5,3), 1,5) \\
& =t(t(3,2, t(1,0,1,4,3), 1,4), 2, t(0,1, \ldots), 1,5) \\
& =t(t(3,2, t(0,1, \ldots), 1,4), 2,1,1,5) \\
& =t(t(3,2,1,1,4), 2,1,1,5) \\
& =t(t(2,1,1,4,3), 2,1,1,5) \\
& =t(t(1,1,4,3,2), 2,1,1,5)=t(1,2,1,1,5)=2,
\end{aligned}
$$

while $u(5,3,2,0,1)=1$.

The true general behavior is somewhat complicated, although (fortunately) the complications do not get worse and worse as $m$ grows larger and larger. Let us define an auxiliary set of functions $g_{j}\left(x_{1}, \ldots, x_{j}\right)$ for $j \geq 2$ as follows:

$$
\begin{aligned}
g_{j}\left(x_{1}, \ldots, x_{j}\right)= & \text { if } j=2 \text { then } x_{2} \\
& \text { else if } x_{1}=x_{2}+1 \text { then } g_{j-1}\left(x_{2}, \ldots, x_{j}\right) \\
& \text { else if } x_{2}=x_{3}+1 \text { then } \max \left(x_{3}, x_{j}\right) \text { else } x_{j} .
\end{aligned}
$$

Theorem 4. The function

$$
\begin{aligned}
f\left(x_{1}, \ldots, x_{m}\right)= & \text { if } x_{1}>\cdots>x_{k} \leq x_{k+1} \text { for some } k \geq 1 \\
& \text { then } g_{k+1}\left(x_{1}, \ldots, x_{k+1}\right) \text { else } x_{1}
\end{aligned}
$$

satisfies then m-dimensional Takeuchi recurrence.

Proof. Given $x_{1}, \ldots, x_{m}$, with $x_{1}>x_{2}$, let

$$
y_{j}=f\left(x_{j}-1, x_{j+1}, \ldots, x_{m}, x_{1}, \ldots, x_{j-1}\right) .
$$

We want to show that $f\left(y_{1}, \ldots, y_{m}\right)=f\left(x_{1}, \ldots, x_{m}\right)$.

If $x_{1}>\cdots>x_{m}$, we have $y_{1}=x_{1}-1$ or $x_{2} ; y_{2}=x_{1}$ or $x_{3} ; \ldots ; y_{m-1}=x_{1}$ or $x_{m}$; and $y_{m}=x_{1}$. We cannot have $y_{1}>\cdots>y_{m}$, because $y_{m}>y_{1}$. Hence there is a unique $k \geq 1$ such that $y_{1}>\cdots>y_{k} \leq y_{k+1}$. And this can happen only if $y_{k+1}=x_{1}$; otherwise $y_{k+1}=x_{k+2}<y_{k}$. It follows that $f\left(y_{1}, \ldots, y_{m}\right)=g_{k+1}\left(y_{1}, \ldots, y_{k+1}\right)=x_{1}=f\left(x_{1}, \ldots, x_{m}\right)$. 
Assume now that $x_{1}>\cdots>x_{k} \leq x_{k+1}$, where $k \geq 2$, and let $a$ be as large as possible such that $x_{i}=x_{i+1}+1$ for $1 \leq i<a$. Then $y_{i}=x_{i+1}$ for $1 \leq i<a$. If $a=k$, we have $y_{k}=x_{k+1}$ and $f\left(y_{1}, \ldots, y_{m}\right)=g_{k}\left(y_{1}, \ldots, y_{k}\right)=g_{k}\left(x_{2}, \ldots, x_{k+1}\right)=g_{k+1}\left(x_{1}, \ldots, x_{k+1}\right)=f\left(x_{1}, \ldots, x_{m}\right)$.

Assume therefore that $a<k$; hence $x_{a}>x_{a+1}+1$. Let $b$ be as large as possible such that $x_{i}>x_{i+1}+1$ for $a \leq i<b$. If $b=k$, we have $y_{i}=x_{k+1}$ for $a \leq i \leq b$, hence $f\left(y_{1}, \ldots, y_{m}\right)=$ $g_{a+1}\left(y_{1}, \ldots, y_{a+1}\right)=g_{a+1}\left(x_{2}, \ldots, x_{a}, x_{k+1}, x_{k+1}\right)=x_{k+1}=g_{k+1}\left(x_{1}, \ldots, x_{k+1}\right)=f\left(x_{1}, \ldots, x_{m}\right)$.

Assume therefore that $b<k$; hence $x_{b}=x_{b+1}+1$ and $y_{b}=x_{b+1}$. Let $z=\max \left(x_{b+1}, x_{k+1}\right)$. We have $y_{i}=z$ for $a \leq i<b-1$. If $a>1$ and $z \geq x_{a}$, we have $f\left(y_{1}, \ldots, y_{m}\right)=g_{a}\left(y_{1}, \ldots, y_{a}\right)=$ $g_{a}\left(x_{2}, \ldots, x_{a}, z\right)=z=g_{k+1}\left(x_{1}, \ldots, x_{k+1}\right)=f\left(x_{1}, \ldots, x_{m}\right)$.

Assume therefore that $a=1$ or $z<x_{a}$. If $x_{b-1}>x_{b}+2$ then $y_{b-1}=z$; otherwise $y_{b-1}=$ $x_{l} \geq x_{k+1}$ for some $l$ in the range $b+1<l \leq k+1$. If $b>a+2$, we have $f\left(y_{1}, \ldots, y_{m}\right)=$ $g_{a+1}\left(y_{1}, \ldots, y_{a+1}\right)=g_{a+1}\left(x_{2}, \ldots, x_{a}, z, z\right)=z=g_{k+1}\left(x_{1}, \ldots, x_{k+1}\right)=f\left(x_{1}, \ldots, x_{m}\right)$. If $b=a+2$, the same chain of equalities is valid unless $x_{b-1}=x_{b}+2$ and $y_{b-1}=x_{l}$ and $x_{l} \neq z$. In the latter case we cannot have $z=x_{k+1}$, for that would imply $x_{k+1} \geq x_{b+1} \geq x_{l}$, hence $x_{l}=x_{k+1}=z$. It follows that $z=x_{b+1}>x_{k+1}$, and $x_{l}<z=y_{b}$. Then $f\left(y_{1}, \ldots, y_{m}\right)=g_{a+2}\left(y_{1}, \ldots, y_{a+2}\right)=$ $g_{a+2}\left(x_{2}, \ldots, x_{a}, z, x_{l}, z\right)=z=f\left(x_{1}, \ldots, x_{m}\right)$.

Assume therefore that $b=a+1$. If $z=x_{k+1}$ we have $x_{k+1} \geq x_{i}$ for $b<i \leq k$, hence $y_{a}=z$ and $y_{i}=x_{i+1}$ or $z$ for $a<i \leq k$. The first appearance of $z$ among $y_{a+1}, \ldots, y_{k+1}$ will show that $f\left(y_{1}, \ldots, y_{m}\right)=z=f\left(x_{1}, \ldots, x_{m}\right)$.

Assume therefore that $z=x_{b+1}>x_{k+1}$. If $x_{a}>x_{b}+2$ then $y_{a}=y_{b}=z$, hence $f\left(y_{1}, \ldots, y_{m}\right)=$ $g_{b}\left(x_{2}, \ldots, x_{a}, z, z\right)=z=f\left(x_{1}, \ldots, x_{m}\right)$.

Assume therefore (and finally) that $x_{a}=x_{b}+2$, so that $y_{a}=x_{l} \geq x_{k+1}$, where $b+1<l \leq k+1$. Then $x_{l}<x_{b+1}=y_{b}$, and $f\left(y_{1}, \ldots, y_{m}\right)=g_{b}\left(x_{2}, \ldots, x_{a}, x_{l}, x_{b+1}\right)=z=f\left(x_{1}, \ldots, x_{m}\right)$. We have proved that $f\left(y_{1}, \ldots, y_{m}\right)=f\left(x_{1}, \ldots, x_{m}\right)$ in all cases.

A machine-based proof of Theorem 4 would be very interesting, especially if it could cope with functions having a variable number of arguments.

Notice that we have not proved that the $m$-dimensional Takeuchi recursion $t\left(x_{1}, \ldots, x_{m}\right)$ actually defines a total function, when $m>3$. We have only shown that $f\left(x_{1}, \ldots, x_{m}\right)$ satisfies the recurrence. If the repeated expansion of $t\left(x_{1}, \ldots, x_{m}\right)$ actually terminates for some sequence of arguments $\left(x_{1}, \ldots, x_{m}\right)$, it must yield the value $f\left(x_{1}, \ldots, x_{m}\right)$; but we have not demonstrated that termination will occur, and there is apparently no obvious ordering on the integer $m$-tuples $\left(x_{1}, \ldots, x_{m}\right)$ that will yield such a proof. Therefore we come to a final question, which will perhaps prove to be the most interesting aspect of the present investigation.

Open Problem 4. Does the $m$-dimensional Takeuchi recursion equation define a total function, for all $m \geq 3$, if it is expanded fully (without call-by-need)? Equivalently, does the recurrence

$$
\begin{aligned}
T\left(x_{1}, \ldots, x_{m}\right)=\text { if } x_{1} \leq & x_{2} \text { then } 0 \\
\text { else } 1 & +T\left(x_{1}-1, x_{2}, \ldots, x_{m}\right)+T\left(x_{2}-1, x_{3}, \ldots, x_{m}, x_{1}\right)+\cdots \\
& +T\left(x_{m-1}-1, x_{m}, x_{1}, \ldots, x_{m-2}\right)+T\left(x_{m}-1, x_{1}, \ldots, x_{m-1}\right) \\
& +T\left(f\left(x_{1}-1, x_{2}, \ldots, x_{m}\right), \ldots, f\left(x_{m}-1, x_{1}, \ldots, x_{m-1}\right)\right)
\end{aligned}
$$


define a total function on the integers $\left(x_{1}, \ldots, x_{m}\right)$, for all $m \geq 3$ ? (Here $f$ is the function of Theorem 4.)

Close inspection of the proof of Theorem 4 implies that a call-by-need technique will always terminate when applied to the recursive equation for $t\left(x_{1}, \ldots, x_{m}\right)$. If $x_{1}>x_{2}>\cdots>x_{k} \leq x_{k+1}$, the values $y_{i}=t\left(x_{i}-1, x_{i+1}, \ldots, x_{i-1}\right)$ need be expanded only for $1 \leq i \leq k+1$, and this will be sufficient to determine the value of $t\left(y_{1}, \ldots, y_{m}\right)=t\left(x_{1}, \ldots, x_{m}\right)$ in a finite number of steps. (The proof is by induction on $k$.) However, the possibility remains that an attempt to expand the "irrelevant" parameters $y_{k+2}, \ldots, y_{m}$ might loop forever. If so, the Takeuchi recurrence would be an extremely interesting example to include in all textbooks about recursion.

\section{References}

[1] N. G. de Bruijn, Asymptotic Methods in Analysis (Amsterdam: North-Holland, 1961).

[2] Richard P. Gabriel, Performance and Evaluation of Lisp Systems (Cambridge, Mass.: MIT Press, 1985).

[3] Ronald L. Graham, Donald E. Knuth, and Oren Patashnik, Concrete Mathematics (Reading, Mass.: Addison-Wesley, 1989).

[4] Donald E. Knuth, Fundamental Algorithms (Reading, Mass.: Addison-Wesley, 1968).

[5] Donald E. Knuth and Herbert S. Wilf, "A short proof of Darboux's lemma," Applied Mathematics Letters 2 (1989), 139-140.

[6] Zohar Manna, Mathematical Theory of Computation (New York: McGraw-Hill, 1974).

[7] Zohar Manna and John McCarthy, "Properties of programs and partial function logic," Machine Intelligence 5 (1970), 27-37.

[8] Zohar Manna and Amir Pnueli, "The validity problem of the 91-function," Stanford Artificial Intelligence Project, Memo No. 68 (August 19, 1968), 20 pp.

[9] Zohar Manna and Amir Pneuli, "Formalization of properties of functional programs," Journal of the ACM 17 (1970), 555-569.

[10] John McCarthy, "An interesting LISP function," unpublished notes, autumn 1978, 3 pp.

[11] John McCarthy and Carolyn Talcott, Lisp: Programming and Proving. Course notes, Computer Science Department, Stanford University, 1980. "Under revision for publication as a book."

[12] J Strother Moore, "A mechanical proof of the termination of Takeuchi's function," Information Processing Letters 9 (1979), 176-181.

[13] I. Takeuchi, "On a recursive function that does almost recusion only," Electrical Communication Laboratory, Nippon Telephone and Telegraph Co., Tokyo, Japan (1978).

[14] Ilan Vardi, "Running time of TAK," preliminary version of unpublished manuscript dated December 1988.

[15] Jean Etienne Vuillemin, Proof techniques for recursive programs, Ph.D. thesis, Stanford University, 1973. 
[16] Jean Vuilllemin, "Correct and optimal implementations of recursion in a simple programming language," Fifth Annual ACM Symposium on Theory of Computing (1973), 224-239.

The preparation of this paper was supported in part by National Science Foundation grant CCR-8610181. 